\title{
Genome-Scale Metabolic Model Based Engineering of Escherichia Coli Enhances Recombinant Single Chain Antibody Fragment Production
}

\section{Aidin Behravan}

Shahid Beheshti University of Medical Sciences School of Pharmacy

Atieh Hashemi ( $\nabla$ at_hashemi@sbmu.ac.ir)

Shahid Beheshti University of Medical Sciences School of Pharmacy https://orcid.org/0000-00017121-5306

\section{Sayed-Amir Marashi}

University of Tehran College of Science

\section{Hamideh Fouladiha}

University of Tehran College of Science

\section{Research Article}

Keywords: AntiEpEX-scFv, Escherichia coli, FVSEOF, Genome-scale metabolic model, glk, Glucokinase.

Posted Date: April 13th, 2021

DOI: https://doi.org/10.21203/rs.3.rs-412220/v1

License: (9) This work is licensed under a Creative Commons Attribution 4.0 International License. Read Full License 


\section{Abstract}

Escherichia coli is an attractive and cost-effective cell factory for producing recombinant proteins such as scFvs. AntiEpEX-scFv is a small antibody fragment receiving considerable attention for the epithelial cell adhesion molecule (EpCAM) targeting. EpCAM is one of the first discovered-cancer-associated biomarkers highly expressed on various types of solid tumors. Hereby, a genome-scale metabolic model guided engineering strategy was proposed to recognize gene targets for improved antiEpEX-scFv production. Flux balance analysis and FVSEOF algorithm identified several potential genetic targets localized in the glucose import system and pentose phosphate pathway that probably guaranteed an improved yield of scFv. Among the targets predicted by the model, $g / k$ gene encoding glucokinase was selected to be overexpressed in the parent strain Escherichia coli BW25113 (DE3). Due to metabolic burden, scFv recombinant expression caused a remarkable decrease in the maximum specific growth rate of the transformed strain. By means of overexpressing $g / k$, presumably increasing carbon flux through the PP pathway, the growth capacity of the E. coli recombinant strain was recovered. Moreover, the engineered strain with $g l k$ overexpression successfully increased scfv production. The titer of antiEpEXscFv reached $235.41 \pm 9.53 \mu \mathrm{g} / \mathrm{mL}(0.428 \mathrm{~g} / \mathrm{g} \mathrm{DCW})$ in the engineered strain compared with the parent strain $(110.236 \pm 7.68 \mu \mathrm{g} / \mathrm{mL} ; 0.202 \mathrm{~g} / \mathrm{g} \mathrm{DCW})$. So, model-based prediction was experimentally validated. This approach can be considered for the improvement of other recombinant proteins production.

\section{Introduction}

Nowadays, majority of clinically valuable proteins are recombinant ones whose production is directly influenced by the metabolism of the producing cell factory. Bacteria and yeasts, are two of the most well established microbial cell factories characterized by high-yield production of recombinant proteins (Ferrer-Miralles and Villaverde 2013). Despite continuous efforts, there are still limiting barriers in overexpression of recombinant proteins that need to be overcome. Metabolic burden observed in producing cells leads to biomass yield reduction, which is part of a stress reaction triggered by protein overexpression and results in reduction of recombinant protein productivity. Circumventing these challenges, metabolic engineering is being successfully employed in developing industrial cell factories (Fernández-Cabezón and Nikel 2020). Using this approach, specific biochemical reactions can be modified by deletion and/or amplification of specific genes leading to regulatory circuits rearrangement (Herrgård et al. 2006). These modifications lead to better productivity of the strains by changing the flux of some metabolic reactions. Based on this strategy, a significant improve in production rate of numerous heterologous products has been reported. For example, heterologous lycopene was produced up to 102 $\mathrm{mg} / \mathrm{L}$ in an engineered Escherichia coli strain. However, the systematical understanding of metabolic pathways as well as regulation mechanisms is highly needed for rational metabolic engineering.

System metabolic engineering strategies that use omics data, have been expanded to guide metabolic engineering. These novel approaches such as genome-scale metabolic model (GEMM)- guided engineering have been evolved building on enormous advances in system biology. Genome-scale modeling can quantitatively predict the cellular behavior at system level and try to recommend genetic 
manipulations to regulate the relationship between target protein overexpression and biomass production so that strains with high growth and protein productivity can be attained. High accuracy in prediction of cell phenotype and minimization of consumed laboratory resources and time for developing productive strains are the most momentous advantages of the model-guided metabolic engineering (Orth et al. 2011). The flux variability analysis (FVA) or the Flux balance analysis (FBA) can be utilized to compute the $E$. coli system model by considering the maximum amount of biomass as an objective target to achieve potential genes for overexpression or down-regulation. FVA or FBA result a flux variability or a flux distribution value respectively. Various algorithms such as FSEOF (flux scanning based on enforced objective flux) (Choi et al. 2010) as a FBA-based method and FVSEOF (flux variability scanning based on enforced objective flux) (Park et al. 2012) and OptForce (Ranganathan et al. 2010) as two FVA-based methods have been employed in the metabolic engineering to determine appropriate genes to be amplified in order to improve industrial strains. In this study, scFv (single chain variable fragment) antibody against EpEX (EpCAM extracellular domain) which has drawn great attention in biomedicine for dual therapeutic and diagnostic applications (Eyvazi et al. 2018), was considered as a model protein to be overproduced in E. coli BW25113 (DE3). The FVSEOF method was used to determine appropriate modifications of the genes in order to increase flux towards antiEpEX-scFv overproduction. Based on the results obtained from in silico FVSEOF analysis, glk gene was selected to be overexpressed. Experimental tests were then performed to evaluate the effect of $g l k$ overexpression on antiEpEX-scFv overproduction.

\section{Materials And Methods}

\section{Metabolic modelling and target gene prediction}

The iJ01366 metabolic model of E. coli (Orth et al. 2011) was used in the COBRA Toolbox v2.0 (Schellenberger et al. 2011) under MATLAB 2014b (Mathworks, USA) with glpk as the solver. The metabolic reaction of antiEpEX-scFv production was added to $i J 01366$ according to the previously described method (under review). FBA was used to determine maximum theoretical amount of antiEpEXscFv $\left(0.034 \mathrm{mg} . g D C W^{-1} \mathrm{~h}^{-1}\right)$ but this value can be achieved only when biomass leads to zero and cannot be used as a realistic constraint for further analysis, therefore FVSEOF method was used by considering a range of 0 to $0.017 \mathrm{mg}_{\mathrm{gDCW}}{ }^{-1} \mathrm{~h}^{-1}$ of antiEpEX-scFv as constraint range in order to achieve maximum biomass. FVSEOF discretizes the range ( 0 to $\left.0.017 \mathrm{mg}_{\mathrm{gDCW}}{ }^{-1} \mathrm{~h}^{-1}\right)$ into five consecutive FVAs and computes the reaction fluxes in the model to maximize biomass production as an objective function. Comparing the result of FVAs declares the reaction fluxes variation, most of the reactions in the model remain unchanged or with less than 0.1 change in flux rate which was ignored. Reactions with significant ascending fluxes are favorable for experimental investigations due to their positive effect on antiEpEXscFv production.

\section{Bacterial strains, plasmids, and cultivation conditions}

Escherichia coli DH5a was used for recombinant plasmid cloning and Escherichia coliBW25113 (DE3) (kindly provided by Prof. Dr. Silke Leimkühler, University of Potsdam, Potsdam, Germany) (Bühning et al. 
2017) was utilized for expression. The plasmid pETDuet-1 (gifted from Dr. Bandehpour, Shahid Beheshti University of Medical Sciences, Tehran, Iran) was utilized as a co-expression vector. The plasmid pETDuet-antiEpEX-scFv was previously constructed in our lab (Behravan and Hashemi 2021). Pfu DNA polymerase was provided from mxcell. T4 DNA ligase, protein molecular weight markers and restriction enzymes were obtained from Thermo Fisher Scientific (USA). DNA fragments were purified from agarose gel using the gel extraction kit (Roche Diagnostics $\mathrm{GmbH}$, Germany). $\mathrm{M} 9$ minimal medium contained (per liter) $0.5 \mathrm{~g}$ of $\mathrm{NaCl}, 6 \mathrm{~g}$ of $\mathrm{Na}_{2} \mathrm{HPO}_{4}, 3 \mathrm{~g}$ of $\mathrm{KH}_{2} \mathrm{PO}_{4}, 1 \mathrm{~g}$ of $\mathrm{NH}_{4} \mathrm{Cl}$ supplemented with $5 \mathrm{~g} / \mathrm{L}$ glucose, $2 \mathrm{mM}$ MgSO4, $0.01 \mathrm{mM} \mathrm{FeCl} 3,0.1 \mathrm{mM} \mathrm{CaCl}$, and $0.1 \mathrm{~mL}$ 1000x trace metals element (Teknova-USA) and LB medium composed of (per liter) $10 \mathrm{~g}$ of tryptone, $5 \mathrm{~g}$ of yeast extract, and $10 \mathrm{~g}$ of $\mathrm{NaCl}$, were used as culture media. All other chemicals were purchased from merck in analytical grade.

\section{Construction of recombinant plasmid}

The glk gene was amplified from the genome of E. coli BW25113 (DE3) employing the primers glk-F, 5CCGGAATTCTGAAGAATGACAAAGTATGC - 3 (the ECoRl site is marked) and glk-R, 5AAACTGCAGCCCGATATAAAAGGAAGGAT - 3 (the Pstl site is marked). Cycling condition was $94^{\circ} \mathrm{C}$ for 3 min followed by 30 cycles of $94^{\circ} \mathrm{C}, 30 \mathrm{~s} ; 56^{\circ} \mathrm{C}, 35 \mathrm{~s}, 72^{\circ} \mathrm{C} 1 \mathrm{~min}$ and $30 \mathrm{~s}$ and 1 cycle of $72^{\circ} \mathrm{C}, 10 \mathrm{~min}$. To generate plasmid pETDuet-glk, the PCR product was digested with restriction enzymes $P s t l, E c o R l$, and then ligated to $p E T D u e t-1$ treated with the same two enzymes. pETDuet-glk-antiEpEX-scFv expression plasmid was constructed by digesting the $\mathrm{pGH}$ vector carrying the antiEpEX-scFv gene with Xhol and Ndel to get the gene with a hexa-histidine tag in its C-Terminal, which was then ligated with pETDuet-glk treated by $\mathrm{Ndel} / \mathrm{XhOl}$. The restriction enzyme digestion assay and sequencing were used to confirm the constructs which were transformed into the chemically competent E. coli BW25113 (DE3) cells for recombinant protein expression.

\section{Expression of antiEpEX-scFv}

For antiEpEX-scFv expression, a single colony of BW25113 (DE3) harboring pETDuet-glk-antiEpEX-scFv was inoculated into $5 \mathrm{~mL}$ of LB medium supplemented with appropriate amounts of ampicillin and incubated for $18 \mathrm{~h}$ at $37^{\circ} \mathrm{C}$ with shaking $(200 \mathrm{rpm})$. After centrifugation $\left(6000 \times \mathrm{g}\right.$ for $5 \mathrm{~min}$ at $\left.4^{\circ} \mathrm{C}\right)$, the pellet was resuspended into $100 \mathrm{~mL}$ of $\mathrm{M} 9$ minimal medium containing ampicillin $(50 \mathrm{mg} / \mathrm{mL})$. When cell density reached an OD $600 \mathrm{~nm}$ of 0.8 , the expression of antiEpEX-scFv was induced with $0.8 \mathrm{mM}$ IPTG at $37^{\circ} \mathrm{C}$. Using centrifugation $\left(10000 \times \mathrm{g}, 10 \mathrm{~min}, 4^{\circ} \mathrm{C}\right)$, cells were harvested after $24 \mathrm{~h}$. For initial determination of protein expression, the cell pellets were suspended in $30 \mathrm{ml}$ of lysis buffer containing 50 $\mathrm{mM}$ Tris $\mathrm{pH} 7.5,1 \mathrm{mM}$ EDTA, $1 \mathrm{mg} / \mathrm{ml}$ lysozyme, $150 \mathrm{mM} \mathrm{NaCl}, 1 \%$ triton X1005 and sonicated for $30 \mathrm{~min}$ (20s ON, 10 s OFF at $400 \mathrm{~W})$. After centrifugation of the cell lysate $\left(10000 \times \mathrm{g}\right.$ for $30 \mathrm{~min}$ at $\left.4^{\circ} \mathrm{C}\right)$, protein samples were electrophoresed on a 15\% SDS-PAGE gel and visualized using Coomassie Brilliant Blue G250 Dye. By using a wet Trasbolt (Bio-Rad, USA), the proteins were electro-transferred from gel into the polyvinylidene difluoride (PVDF) membrane to perform western blot analysis. Transferred membrane was blocked in $5 \%$ nonfat milk for 1 hour and then was washed three times with TBST and then incubated in His-tag antibody (Sigma, UK) overnight. After washing again, the membrane was incubated in anti-mouse HRP conjugated immunoglobulin (Sigma, UK) as secondary antibody for two hours and then detected by 
means of a solution of 3,3'-Diaminobenzidine DAB (Sigma, UK). The recombinant antiEpEX-scFv was purified using the Ni-NTA affinity chromatography column under denaturing conditions based on the manufacturer's protocol (Qiagen, Netherlands). Utilizing the bicinchoninic acid assay (BCA assay), the concentration of the purified protein was measured (Takara BCA Protein Assay Kit, Takara, Japan).

\section{Growth profile and glucose analysis}

To investigate cell growth profile, optical density at $600 \mathrm{~nm}$ was determined every hour, using spectrophotometer (E-Chrome Tech, Taipei, Taiwan). Logarithmic derivation of the optical density curve was used for calculation of growth rate. In order to determine the glucose concentration, one milliliter of sample from culture broth was harvested in 1 hour intervals. The supernatant was collected following centrifugation at $10000 \times \mathrm{g}(10 \mathrm{~min})$. The concentration of glucose was measured using a commercial enzymatic kit (Megazyme, Wicklow, Ireland).

\section{Real-time PCR analysis}

The relative expression of $g / k$ as a target gene was compared between E. coli BW25113/Duet-glk-scFv, wild type strain E. coli BW25113 and E. coli BW25113/Duet-scFv using RT-qPCR. E. coli strains were cultured in $50 \mathrm{ml}$ of $\mathrm{M} 9$ medium and induced with $0.8 \mathrm{mM} \mathrm{IPTG}$ in $\mathrm{OD}_{600}=0.8$. After 3 hours, samples were collected and diluted to $\mathrm{OD}_{600}=0.4$. Based on the manufacturer's protocol, total RNA was extracted from bacterial cells utilizing Trizol reagent (Ambion). The purity and quantity of the isolated RNA were measured by Synergy HTX multimode reader (BioTek) and was stored at $-80^{\circ} \mathrm{C}$ for further use. CDNA synthesis kit (YT450; Yekta Tajhiz Azma) was employed to synthesize cDNA according to the instruction provided by the manufacturer. Reviewing the literature, primers were selected and assessed for GC content, specificity, secondary structures, and amplicon size. Primers sequences synthesized by Metabion are presented in Table1. StepOne Real-Time PCR System (Applied Biosystems) was employed for SYBRGreen qPCR reactions in 48 well optical reaction plates. CDNA ( $0.5 \mathrm{ng} /$ reaction) was used as a template for qPCR reactions with $5 \mu$ S SYBR Green PCR Master Mix (2x) (YT2551; Yekta Tajhiz Azma) and primers at $10 \mu \mathrm{M}$ final concentration. Samples were exposed to thermal plan as follows: $95^{\circ} \mathrm{C}, 30 \mathrm{~s}$ followed by 40 cycles of $95^{\circ} \mathrm{C}, 5 \mathrm{~s}$ and $60^{\circ} \mathrm{C}, 30 \mathrm{~s}$. The PCR reactions were done in three technical replicates for more accuracy. $2^{-\Delta \Delta \mathrm{Ct}}$ method was used to evaluate relative gene expression against the reference gene.

Table 1

Sequences of RT-qPCR primers

\begin{tabular}{|ll|}
\hline Name & oligonucleotide Sequences $\left(5^{\square} \mathbf{3}<-{ }^{\natural}\right)$ \\
\hline $16 S$ & F: TACCGCATAACGTCGCAAGA \\
& R: AGTCTGGACCGTGTCTCAGT \\
glk & F: CTGTATTGCCATCGCTTGCC \\
& R: TTACCTTCGACCGGTTCTGC \\
\hline
\end{tabular}




\section{Results}

\section{Prediction of overexpression targets}

E. coli GEMM named iJ01366 was employed for prediction of metabolic engineering targets which can improve antiEpEX-scFv production. FVSEOF predicted ten metabolic reactions to enhance antiEpEX-scFv productivity via their overexpression. Two genes related to the importation of glucose to the cell (ga/P and $g / k$ ), four genes related to the pentose phosphate pathway (PPP) ( $z w f, r p e, p g l$, gnd), two genes related to the folate biosynthesis pathway (focA and purU), and two genes related to alternative carbon metabolism sub-system ( $x y / \mathrm{A}$, mak) were suggested by FVSEOF analysis for overexpression. These reactions in metabolic pathway are illustrated in Fig. 1.

The transcriptome analysis showed that the glk gene was upregulated during the recombinant protein production in E. coli (Oh and Liao 2000). Therefore, in this study the glk gene was selected as the target gene for overexpression.

\section{Evaluation of glk transcription level}

In order to validate the overexpression of $g / k$, real-time PCR experiment was employed. After $3 \mathrm{~h}$ of induction, the relative quantification of $g l k$ transcript revealed that $g / k$ gene was upregulated in $E$. coli BW25113/Duet-glk-scFv by 14.78 -fold and 925.56 -fold in comparison to the $E$. coli BW25113/Duet-scFv and the parent strain respectively.

\section{The effect of glk overexpression on antiEpEX-scFv production}

Glucokinase gene was amplified from E. coli BW25113 (DE3) genome by PCR reaction resulting in a 966 bp glk gene which can encode 321 amino acids with the molecular weight of about $35 \mathrm{kDa}$. glk and antiEpEX-scFv coding sequences were inserted into the first and second multiple cloning sites (MCS) of pETDuet-1 respectively to generate the plasmid pETDuet-glk-antiEpEX-scFv as shown in Fig. 2. The constructed pETDuet-glk-antiEpEX-scFv plasmid was confirmed by restriction enzyme digestion (Fig. 3) and sequencing and transformed into E. coli BW25113 (DE3). Recombinant E. coli BW25113/Duet-glkscFv and $E$. coli BW25113/Duet-scFv were cultured in M9 minimal medium containing a suitable antibiotic to an $\mathrm{OD}_{600}$ of 0.8 and induced with $0.8 \mathrm{mM}$ IPTG for $24 \mathrm{~h}$. The SDS-PAGE analysis exhibited the presence of two separate protein bands with molecular weights of about $35 \mathrm{kDa}(g / k)$ and about 29 $\mathrm{kDa}$ (antiEpEX-scFv) (Fig. 4a). The expressed scFv protein was confirmed using western blot analysis and an antibody against a C-terminal histidine tag (6xHis-tag) (Fig. 4b). Recombinant antiEpEX-scFv protein was purified through affinity chromatography using Ni-NTA matrix (Fig. 4c). BCA analysis was used to calculate recombinant protein concentration. According to the concentration of the purified antiEpEXscFv, the $g l k$-overexpressed strain showed an increase in antiEpEX-scFv titer $(235.41 \pm 9.53 \mu \mathrm{g} / \mathrm{mL} ; 0.428$ $\mathrm{g} / \mathrm{g} \mathrm{DCW}$ ), which was approximately 2.135 times higher than that in strain with no glk overexpression $(110.236 \pm 7.68 \mu \mathrm{g} / \mathrm{mL} ; 0.202 \mathrm{~g} / \mathrm{g} \mathrm{DCW})$ after $24 \mathrm{~h}$ post-induction cultivation (Fig. $4 \mathrm{~b})$. The results 
suggested that the altered glucose metabolism by $g / k$ overexpression could improve the antiEpEX-scFv production.

\section{Growth and glucose consumption profiles}

To examine how co-expression of $g / k$ with antiEpEX-scFv can affect bacterial growth rate and glucose consumption rate, wild-type strain (BW25113), recombinant E. coli BW25113/Duet-glk-scFv and E. coli BW25113/Duet-scFv, were cultured in M9 minimal medium containing 5 or $10 \mathrm{~g} / \mathrm{L}$ glucose at $37^{\circ} \mathrm{C}$. IPTG in final concentration of $0.8 \mathrm{mM}$ was added in $\mathrm{OD}_{600}=0.8$ for protein induction. In order to obtain growth profile, OD in $600 \mathrm{~nm}$ were measured for $24 \mathrm{~h}$ with 1 -hour intervals. Each measurement was performed in duplicate.

All strains in M9 minimal medium containing $5 \mathrm{~g} / \mathrm{L}$ glucose (Fig. $5 \mathrm{a}$ and $5 \mathrm{~b}$ ), grow logarithmically as long as glucose is available and when glucose is depleted, enter to the stationary phase (Fig. 5a). As shown in Fig. 5a, maximum specific growth rate in recombinant E.coli BW25113/Duet-scFv and BW25113/Duetglk-scFv $\left(\mu_{\max }=0.462 \pm 0.034\right.$ and $\mu_{\max }=0.552 \pm 0.003$ respectively $)$ was lower than that in the parent strain $\left(\mu_{\max }=0.637 \pm 0.013\right)$. Since recombinant protein production and cell growth share some common precursors, increased protein expression at the expense of decreased cell density maybe due to alteration of intracellular fluxes through the biomass precursors towards protein synthesis in recombinant strains. However, recombinant $E$. coli BW25113/Duet-glk-scFv has greater specific growth rate than the $E$. coli BW25113/Duet-scFv (Table 2) especially when more glucose is available in the media. As shown in Fig. $5 \mathrm{C}$ and presented in Table $2, \mu_{\max }$ is much higher for E. coli BW25113/Duet-glk-scFv $(0.81 \pm 0.043)$ in comparison to $E$. coli BW25113/Duet-scFv $(0.592 \pm 0.003)$ and wild type strain $(0.729 \pm 0.022)$. As expected, maximum specific growth rate of all strains in M9 minimal medium containing $10 \mathrm{~g} / \mathrm{L}$ glucose, is higher than that in the medium supplemented with $5 \mathrm{~g} / \mathrm{L}$ glucose.

Table 2

Specific growth rate of different strains in M9 minimal medium in different glucose concentrations

\begin{tabular}{|lll|}
\hline Strains & $\begin{array}{l}\text { Growth rate }\left(\mathrm{h}^{-1}\right) \text { in } \mathbf{M 9}+\mathbf{5} \mathbf{~ g / L} \\
\text { glucose }\end{array}$ & $\begin{array}{l}\text { Growth rate }\left(\mathrm{h}^{-1}\right) \text { in } \mathbf{M 9}+\mathbf{1 0} \mathbf{~ g / L ~} \\
\text { glucose }\end{array}$ \\
\hline BW25113 & $0.637 \pm 0.013$ & $0.729 \pm 0.022$ \\
\hline BW25113-Duet-scFv & $0.462 \pm 0.034$ & $0.592 \pm 0.003$ \\
\hline $\begin{array}{l}\text { BW25113-Duet-glk- } \\
\text { ScFv }\end{array}$ & $0.552 \pm 0.003$ & $0.81 \pm 0.043$ \\
\hline
\end{tabular}

As shown in the Table 3, glucose consumption rate in E. coli BW25113/Duet-glk-scFv (1.145 \pm 0.01$)$ is greater than that in the $E$. coli BW25113/Duet-scFv $(0.968 \pm 0.02)$ and wild type strain $(0.851 \pm 0.05)$. Also, as illustrated in Fig. $5 b$, the required time for the complete consumption of glucose for parental strain and the E. coli BW25113/Duet-glk-scFv were 10 hours while E. coli BW25113/Duet-scFv needs two more hours to consume all the glucose in the medium. Interesting result from Fig. $5 \mathrm{~d}$ is that the required time for complete consumption of glucose for parental strain and E. coli BW25113/Duet-glk-scFv is similar 
(about $24 \mathrm{~h}$ ) while E. coli BW25113/Duet-scFv doesn't consume all of the available glucose in the medium up to $24 \mathrm{~h}$ (Fig. $5 \mathrm{~d}$ ). It confirmed that the overexpressed glk gene functioned well in $E$. coli BW25113/Duet-glk-scFv.

Table 3

Glucose consumption rate in different strains in M9 minimal medium in different glucose concentrations

\begin{tabular}{|c|c|c|}
\hline Strains & $\begin{array}{l}\text { uptake rate }(\mathrm{g} / \mathrm{g} \mathrm{DCW} \mathrm{h}) \text { in } \mathrm{M} 9+5 \\
\mathrm{~g} / \mathrm{L} \text { glucose }\end{array}$ & $\begin{array}{l}\text { uptake rate }(\mathrm{g} / \mathrm{g} \mathrm{DCW} \mathrm{h}) \text { in } \mathrm{M} 9+10 \\
\mathrm{~g} / \mathrm{L} \text { glucose }\end{array}$ \\
\hline BW25113 & $0.851 \pm 0.05$ & $0.730 \pm 0.034$ \\
\hline $\begin{array}{l}\text { BW25113-Duet- } \\
\text { scFv }\end{array}$ & $0.968 \pm 0.02$ & $0.706 \pm 0.039$ \\
\hline $\begin{array}{l}\text { BW25113-Duet- } \\
\text { glk-scFv }\end{array}$ & $1.145 \pm 0.01$ & $1.175 \pm 0.03$ \\
\hline
\end{tabular}

As a conclusion, the enhancement of the growth rate and the glucose consumption rate by overexpressing $g l k$ gene is considerable in the results.

\section{Discussion}

Development of the hosts that have desirable metabolic phenotypes and ability to produce heterologous products is an important issue in microbial metabolic engineering. Utilizing various algorithms, GEMM based approaches enabled scientists to recognize gene deletion or overexpression targets for developing cell factories. For example, using MOMA simulations, L-valine biosynthesis was successfully improved in an engineered $E$. coli strain (Park et al. 2007). Also, amplification of idi gene selected by FSEOF together with the $d x s$ gene led to lycopene overproduction (Choi et al. 2010). However, metabolic phenotypes prediction after gene deletion is much simpler than that after gene amplification. Because the corresponding metabolic fluxes of the deleted gene can be assumed as zero, while, owing to complex regulation of the metabolic network, the corresponding fluxes of the amplified genes do not certainly increase. Moreover, the amount of increase in metabolic fluxes corresponding to the gene amplification is difficult to be predicted. In this study, in order to increase the flux towards antiEpEX-scFv overproduction, the $g l k$ gene was selected for amplification among the several targets predicted by FVSEOF.

According to our results, recombinant expression of scFv and glk resulted in a decrease in the maximum specific growth rate of the recombinant strains compared with the parent strain. A decrease in the growth rate is normally detectable in bacteria transformed with multicopy plasmids to produce a recombinant protein. Actually, plasmid DNA replication, plasmid-encoded mRNA synthesis and translation in bacteria frequently place a metabolic burden into the engineered strains that usually results in growth retardation (Flores et al. 2004). This metabolic burden may be due to the cell inability to supply the extra demand of energy and building blocks required for plasmid replication and foreign multicopy genes expression ( $\mathrm{Li}$ and Rinas 2020). However, a significant increase was observed in the $\mu_{\max }$ of the recombinant strains from $0.592 \pm 0.003$ in BW25113-Duet-scFv to $0.81 \pm 0.043$ in BW25113-Duet-glk-scFv when the expression of the $g / k$ gene was increased which was comparable to the wild-type strain $(0.729 \pm 0.022)$. glk gene 
encodes the enzyme glucokinase catalyzing the ATP-dependent phosphorylation of the glucose that was imported by GalP. glk overexpression probably compensates the special metabolic demands of the engineered strains via increasing the carbon flux into the PP pathway. The PP pathway which is closely interconnected with glycolysis normally provides some of the required blocks for biosynthesis of histidine, nucleotide and aromatic amino acids e.g., erythrose-4-phosphate (E4P) and ribose-5- phosphate (R5P) (Stincone et al. 2015). Also NADPH, a power of biosynthetic reactions, was reduced in its oxidative branch (Christodoulou et al. 2018). In a similar study, engineering of the pentose phosphate pathway led to reduction of the metabolic load caused by the recombinant protein production (Flores et al. 2004). Moreover, using this approach, a significant positive effect was observed on the productivity of scFv producing strains in the current study. The glk-overexpressed strain produced approximately 2.135 times higher titer of ScFv than the strain with no glk overexpression. So, the metabolic engineering target predicted in our study was validated via the improvement observed in the scFv production.

Recently, the integration of the computational methods and omics at the systems level can empower metabolic engineering. Employing DNA microarray, oh et al revealed that the overproduction of recombinant non-toxic LuxA could lead to the downregulation of $p p c$, fba, gnd, and atpA genes as well as upregulation of heat shock and $g / k$ genes in E. coli strains including JM109, MC4100, and VJS676A. Based on the transcriptome profile obtained in oh et al study, glucose kinase might have the major role instead of the phosphotransferase system (PTS) to provide glucose6-phosphate in protein overproducing condition in the E. coli cells (Oh and Liao 2000). On the other hand, overexpression of the recombinant proteins was shown to induce heat shock genes and rapid stress response. Interestingly, $g / k$ has been reported to play an essential role in bacterial stress responses. Although, this gene plays a minor role in glucose metabolism, but under stress condition like heterologous protein expression or growth in acidic condition, this glycolysis enzyme is required to supply sufficient level of glucose6-phosphate (Arora and Pedersen 1995; Zhang et al. 2020). So, glk seems to be a suitable target gene to be overexpressed to achieve increased recombinant protein productivity, which is consistent with our results.

Here, the GEMM-guided metabolic engineering strategy was used to improve the scFv production in Escherichia coli BW25113 (DE3). The engineered strain with glk overexpression successfully increased scFv production. The titer of antiEpEX-scFv reached $235.41 \pm 9.53 \mu \mathrm{g} / \mathrm{mL}(0.428 \mathrm{~g} / \mathrm{g} \mathrm{DCW})$ in the engineered strain compared with the parent strain $(110.236 \pm 7.68 \mu \mathrm{g} / \mathrm{mL} ; 0.202 \mathrm{~g} / \mathrm{g} \mathrm{DCW})$. Our method for the production of scFv is a successful example which can be considered for the improvement of other recombinant proteins production.

\section{Declarations}

\section{Funding}

This work was supported by the research deputy of Shahid Beheshti University of Medical Sciences in Tehran, Iran (IR.SBMU.PHARMACY.REC.1399.372).

\section{Conflicts of interest/Competing interests}


We have no conflict of interest to declare

\section{Availability of data and material}

Not applicable

Code availability

Not applicable

\section{Authors' contributions}

$\mathrm{AH}$ and SAM have made substantial contributions to conception and design. $\mathrm{AB}$ and $\mathrm{HF}$ carried out the experiments and analyzed the data. $A B$ wrote the manuscript. $A H$ reviewed and edited the article for spelling, grammar, and intellectual content. $\mathrm{AH}$ and SAM organized and supervised the whole project. $\mathrm{AH}$ provided the facilities and materials required for the project. All authors agreed to be responsible for the content of the work.

\section{Ethics approval}

Not applicable

\section{Consent to participate}

Not applicable

\section{Consent for publication}

All authors have approved the manuscript and agree with its submission to the World Journal of Microbiology and Biotechnology.

\section{Acknowledgements}

We thanked Prof. Dr. Silke Leimkühler and Dr. Bandehpour for providing Escherichia coli BW25113 (DE3) and plasmid pETDuet-1 respectively.

\section{References}

1. Arora K, Pedersen P (1995) Glucokinase of Escherichia coli: induction in response to the stress of overexpressing foreign proteins. Arch Biochem Biophys 319 2:574-578. https://doi.org/10.1006/abbi.1995.1333

2. Behravan A, Hashemi A (2021) Statistical optimization of culture conditions for expression of recombinant humanized anti-EpCAM single-chain antibody using response surface methodology. Res Pharm Sci 16:153-164. https://doi.org/10.4103/1735-5362.310522 
3. Bühning M, Friemel M, Leimkühler S (2017) Functional Complementation Studies Reveal Different Interaction Partners of Escherichia coli IscS and Human NFS1. Biochemistry 56:4592-4605. https://doi.org/10.1021/acs.biochem.7b00627

4. Choi HS, Lee SY, Kim TY, Woo HM (2010) In silico identification of gene amplification targets for improvement of lycopene production. Appl Environ Microbiol 76:3097-3105. https://doi.org/10.1128/AEM.00115-10

5. Christodoulou D, Link H, Fuhrer T et al (2018) Reserve Flux Capacity in the Pentose Phosphate Pathway Enables Escherichia coli's Rapid Response to Oxidative Stress. Cell Syst 6:569-578.e7. https://doi.org/10.1016/j.cels.2018.04.009

6. Eyvazi S, Farajnia S, Dastmalchi S et al (2018) Antibody Based EpCAM Targeted Therapy of Cancer, Review and Update. Curr Cancer Drug Targets 18:857-868. https://doi.org/ $10.2174 / 1568009618666180102102311$

7. Fernández-Cabezón L, Nikel PI (2020) Chap. 11 - Advanced metabolic engineering strategies for the development of sustainable microbial processes. In: New and Future Developments in Microbial Biotechnology and Bioengineering. Elsevier, pp 225-246. https://doi.org/10.1016/B978-0-44464301-8.00011-1

8. Ferrer-Miralles N, Villaverde A (2013) Bacterial cell factories for recombinant protein production; expanding the catalogue. Microb Cell Fact 12:113. https://doi.org/10.1186/1475-2859-12-113

9. Flores S, de Anda-Herrera R, Gosset G, Bolívar FG (2004) Growth-rate recovery of Escherichia coli cultures carrying a multicopy plasmid, by engineering of the pentose-phosphate pathway. Biotechnol Bioeng 87:485-494. https://doi.org/10.1002/bit.20137

10. Herrgård MJ, Lee BS, Portnoy V, Palsson B (2006) Integrated analysis of regulatory and metabolic networks reveals novel regulatory mechanisms in Saccharomyces cerevisiae. Genome Res 16:627635. https://doi.org/10.1101/gr.4083206

11. Li Z, Rinas U (2020) Recombinant protein production associated growth inhibition results mainly from transcription and not from translation. Microb Cell Fact 19:83. https://doi.org/10.1186/s12934020-01343-y

12. Oh M-K, Liao JC (2000) DNA Microarray Detection of Metabolic Responses to Protein Overproduction in Escherichia coli. Metab Eng 2:201-209. https://doi.org/10.1006/mben.2000.0149

13. Orth JD, Conrad TM, $\mathrm{Na}$ J et al (2011) A comprehensive genome-scale reconstruction of Escherichia coli metabolism-2011. Mol Syst Biol 7:535. https://doi.org/10.1038/msb.2011.65

14. Park JH, Lee KH, Kim TY, Lee SY (2007) Metabolic engineering of Escherichia coli for the production of $L$-valine based on transcriptome analysis and in silico gene knockout simulation. Proc Natl Acad Sci U S A 104:7797-7802. https://doi.org/10.1073/pnas.0702609104

15. Park JM, Park HM, Kim WJ et al (2012) Flux variability scanning based on enforced objective flux for identifying gene amplification targets. BMC Syst Biol 6:106. https://doi.org/10.1186/1752-0509-6106 
16. Ranganathan S, Suthers PF, Maranas CD (2010) OptForce: An optimization procedure for identifying all genetic manipulations leading to targeted overproductions. PLoS Comput Biol 6: https://doi.org/10.1371/journal.pcbi.1000744

17. Schellenberger J, Que R, Fleming RMT et al (2011) Quantitative prediction of cellular metabolism with constraint-based models: the COBRA Toolbox v2.0. Nat Protoc 6:1290-1307. https://doi.org/10.1038/nprot.2011.308

18. Stincone A, Prigione A, Cramer T et al (2015) The return of metabolism: biochemistry and physiology of the pentose phosphate pathway. Biol Rev 90:927-963. https://doi.org/10.1111/brv.12140

19. Zhang W, Chen X, Sun W et al (2020) Escherichia coli Increases its ATP Concentration in Weakly Acidic Environments Principally through the Glycolytic Pathway. Genes (Basel) 11:991. https://doi.org/10.3390/genes11090991

\section{Figures}

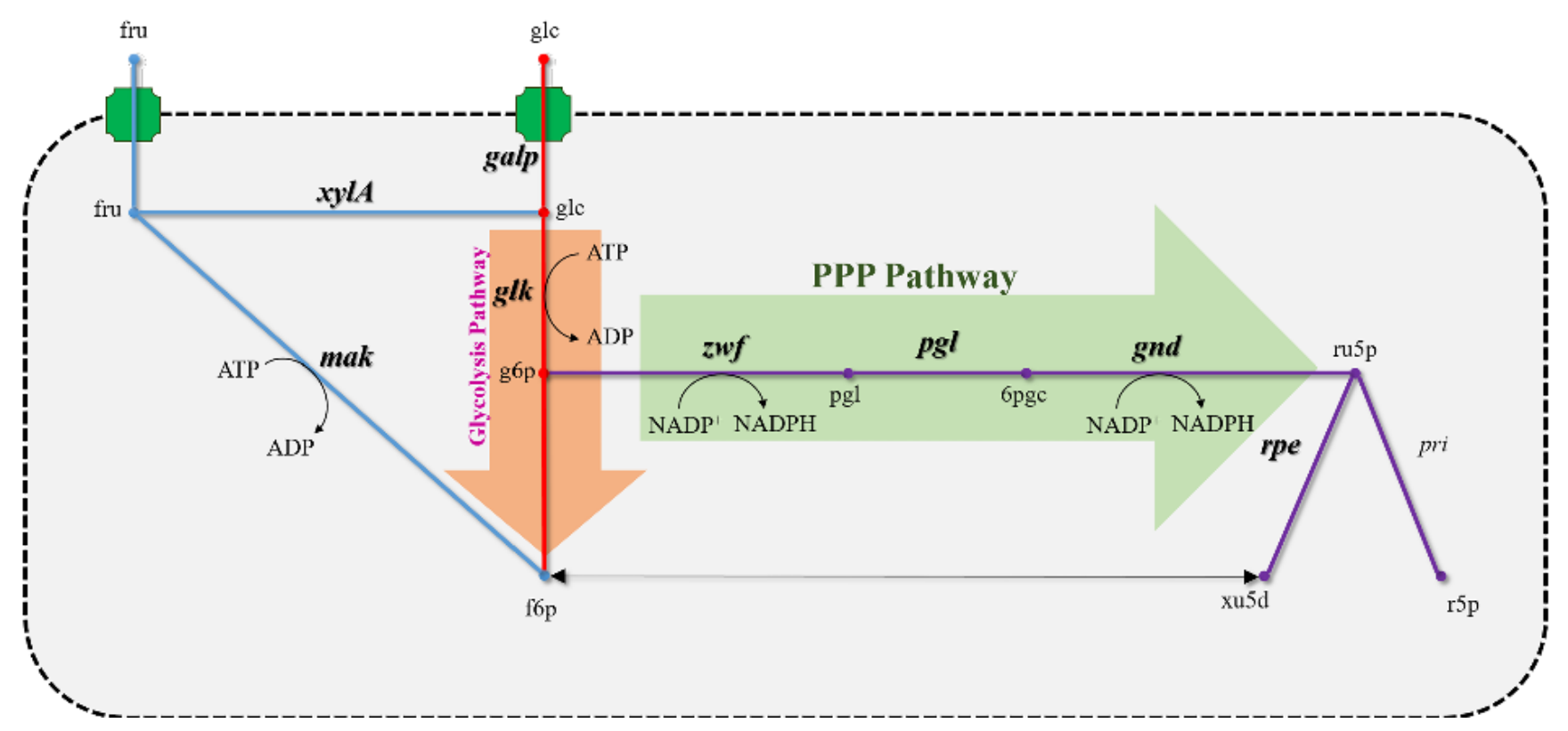

\section{Figure 1}

Overexpression targets illustrated in the metabolic map. The predicted targets for overexpression are indicated by their bold gene names.(PPP pathway: Pentose Phosphate Pathway, fru: Fructose, glc: Glucose, g6p: glucose-6-phosphate, f6p: Fructose-6-phosphate, pgl: 6-phosphogluconolacton, Ru5P: ribulose-5-phosphate, 6pgc: 6-phosphogluconate, R5P: ribose-5-phosphate, galP: galactose permease, zwf: glucose-6-phosphate dehydrogenase, xylA: xylose Isomerase, mak: fructokinase, glk: glucokinase, gnd: 6-phosphogluconate dehydrogenase, pgl: 6-phosphogluconolactonase, rpe: ribulose-5-phosphate-3epimerase) 


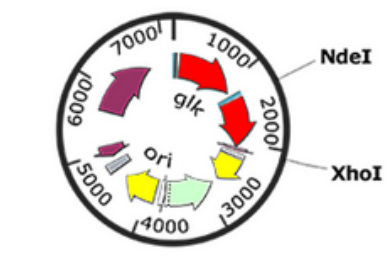

pETDuet-glk-antiEpEX-scFv $7150 \mathrm{bp}$

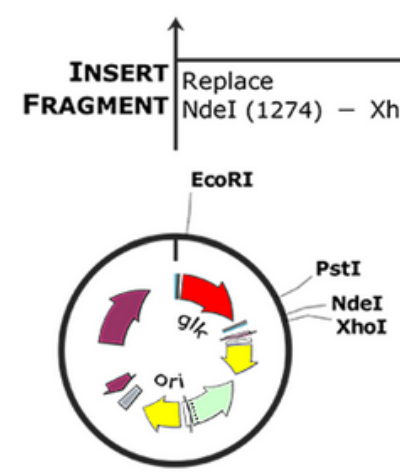

pETDuet-glk

$6396 \mathrm{bp}$

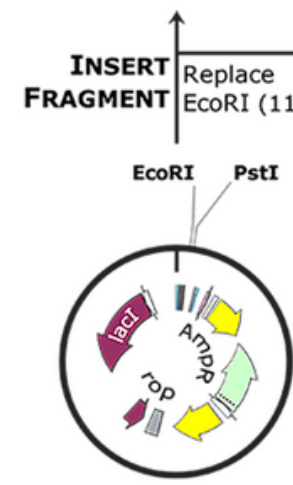

PETDuet-1

$5420 \mathrm{bp}$

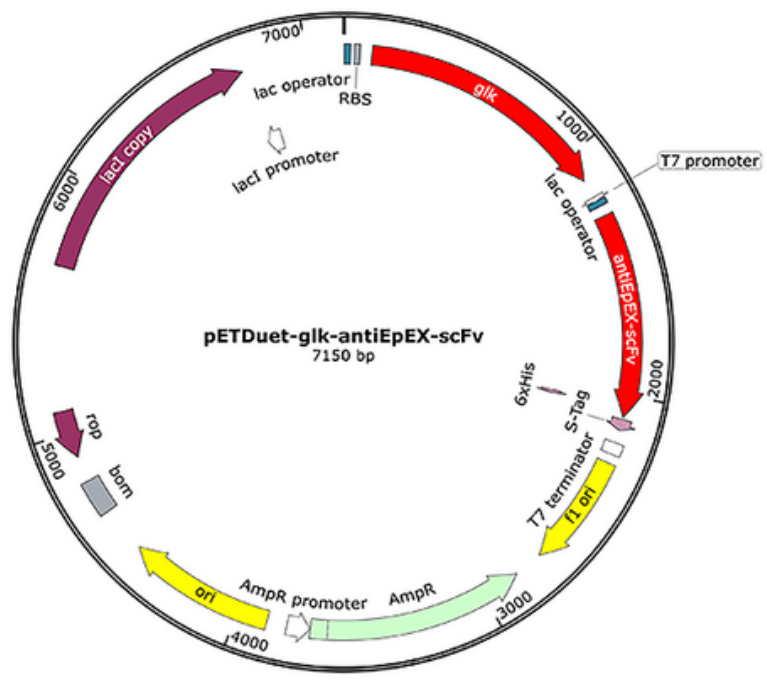

Insert

NdeI (2) - XhoI (812)

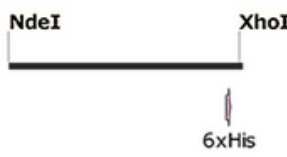

antiEpEX-scFv

$817 \mathrm{bp}$

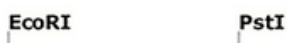

$\underset{1004 \text { bp }}{\text { glk }}$

\section{Figure 2}

History and construction of the pETDuet-glk-antiEpEX-scFv expression vector: gene encoding glk was cloned between EcoRI and Pstl, antiEpEX-scFv gene was cloned between Ndel and Xhol sites of pETDuet -1. Maps were designed with SnapGene Version 5.2.4 


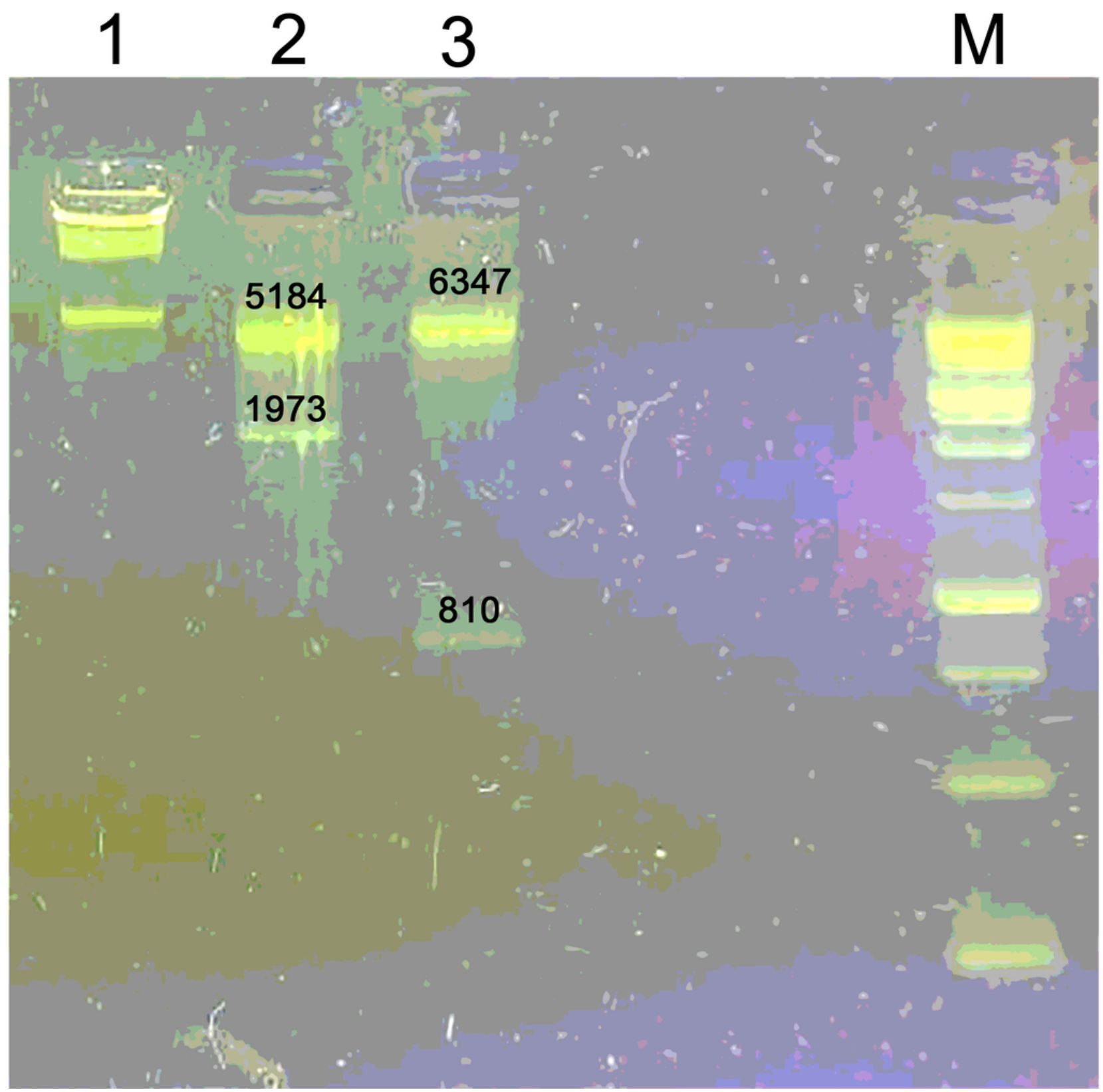

\section{Figure 3}

Confirmation of the recombinant plasmid pETDuet-glk-antiEpEX-ScFv by enzymatic digestion reaction: 1: plasmid pETDuet-glk-antiEpEX-scFv (7150bp); 2: recombinant plasmid pETDuet-glk-antiEpEX-scFv digested with EcoRI and Pstl (6347bp and 1973bp); 3: recombinant plasmid pETDuet-glk-antiEpEX-scFv digested with Xhol and Ndel (5184bp and 810bp); M: DNA marker 1kb. 
a
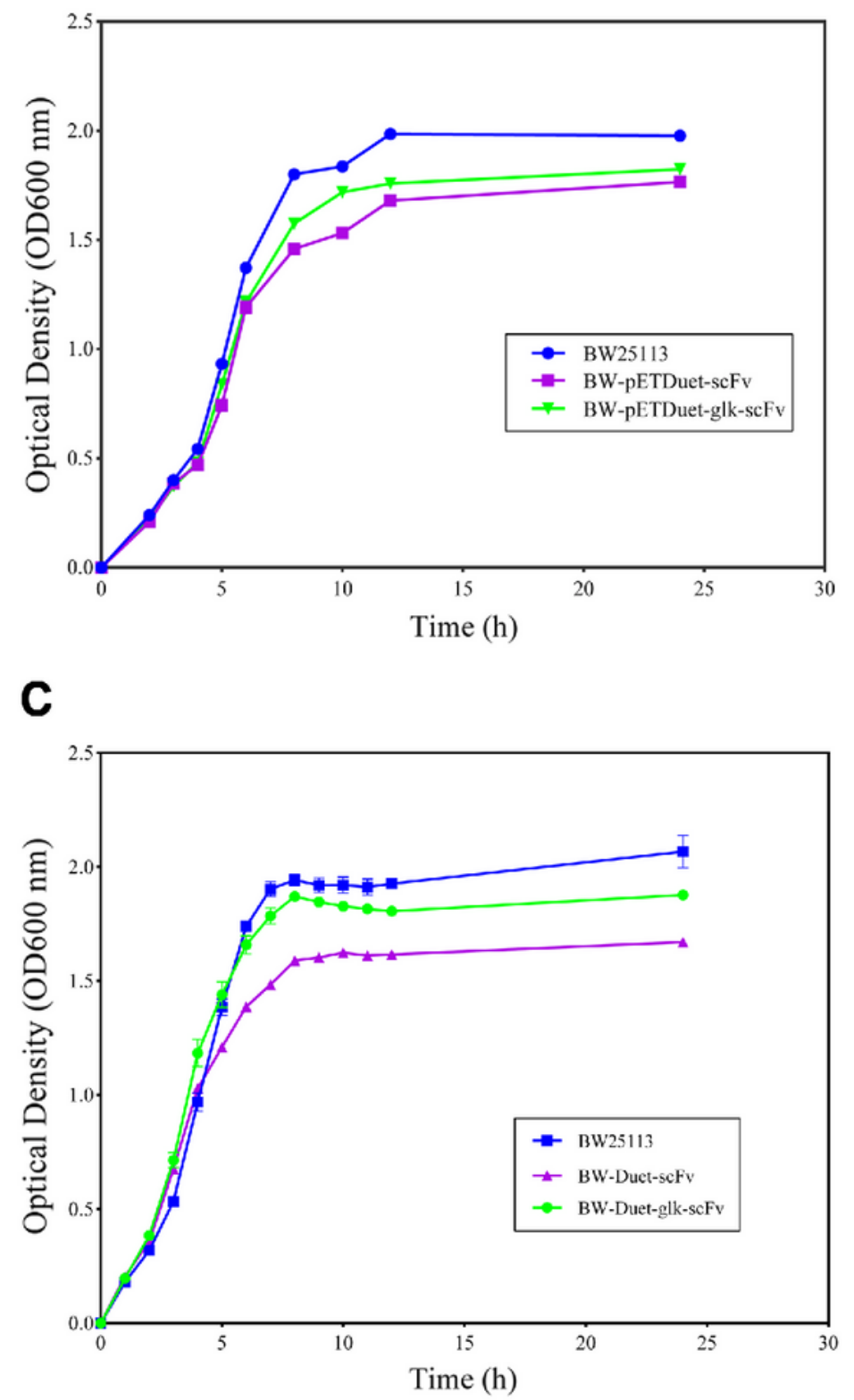

b
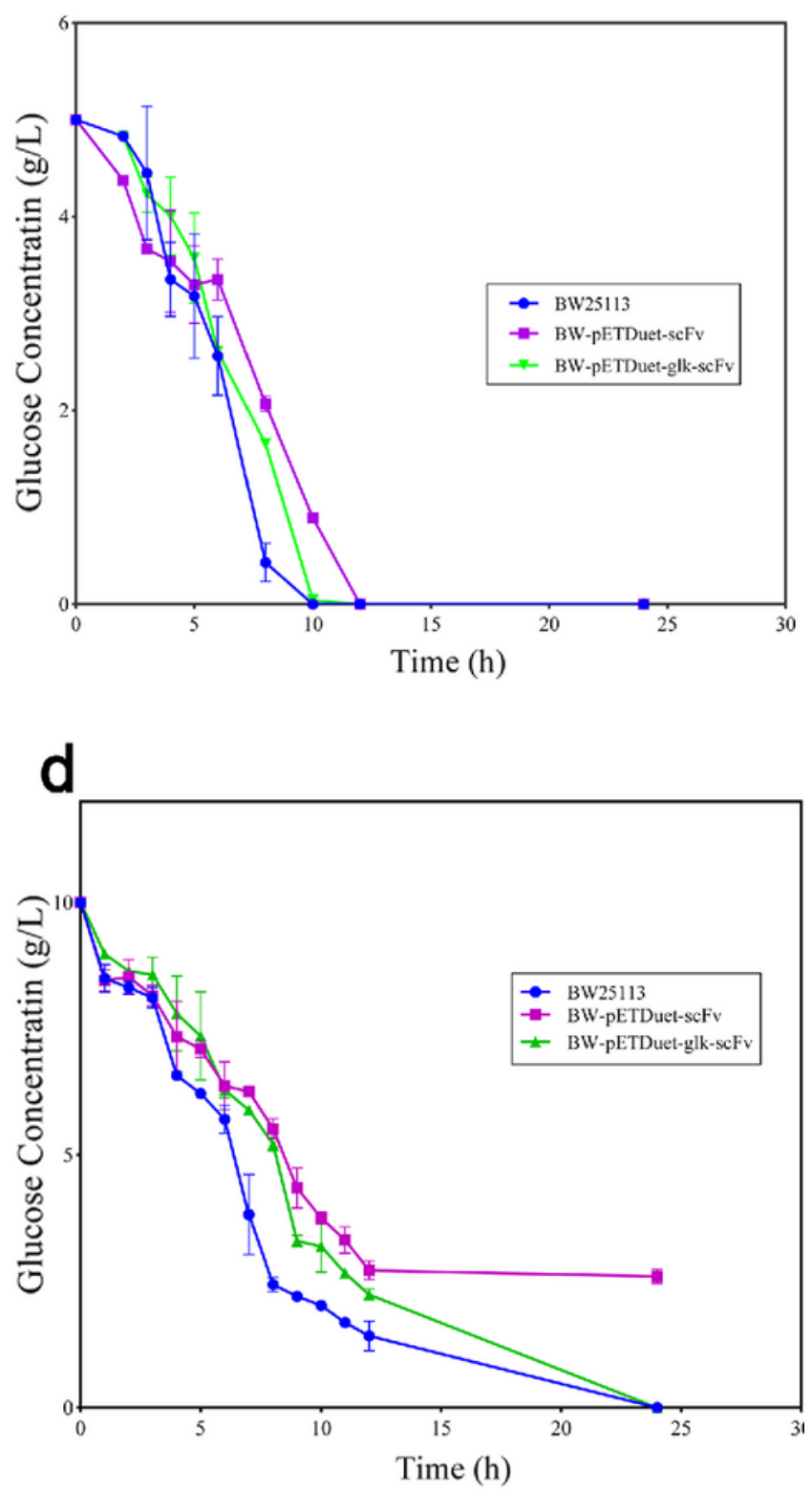

Figure 4

SDS-PAGE and western blot analyses for characterization of the antiEpEX-scFv recombinant protein in E. coli cell extracts. A: SDS-PAGE analysis of total lysate. $(1,2)$ : Induced total lysate of BW25113/DuetScFv; (M): Molecular weight protein marker (14.4-116 kDa); (3,4): Induced bacterial lysate with the empty plasmid pETDuet-1, $(5,6)$ : Induced total lysate of E. coli BW25113/Duet-glk-scFv. B: Western blot analysis for the recombinant antiEpEX-scFv using anti-polyhistidine monoclonal antibody. (C-): Uninduced bacterial lysate; (M): Prestained molecular weight marker (10 - $250 \mathrm{kDa})$; (1): Induced total lysate of BW25113/Duet- scFv (2): Induced total lysate of E. coli BW25113/Duet-glk-scFv C: SDS-PAGE analysis of the purified antiEpEX-scFv. (M): Molecular weight protein marker (14.4 - $116 \mathrm{kDa})$; (C-) Uninduced bacterial lysate. (1) Eluted protein fraction from E. coli BW25113/Duet-scFv; (2) Eluted protein fraction from E. coli BW25113/Duet-glk-scFv. All experiments were done in M9 minimal medium 
containing glucose as a carbon source. When OD600 reached to 0.8 , cells were induced for $24 \mathrm{~h}$ using 0.8 mM IPTG. The experiments were performed in duplicates. The protein bands corresponded to antiEpEXscFv and glk are shown by arrows.
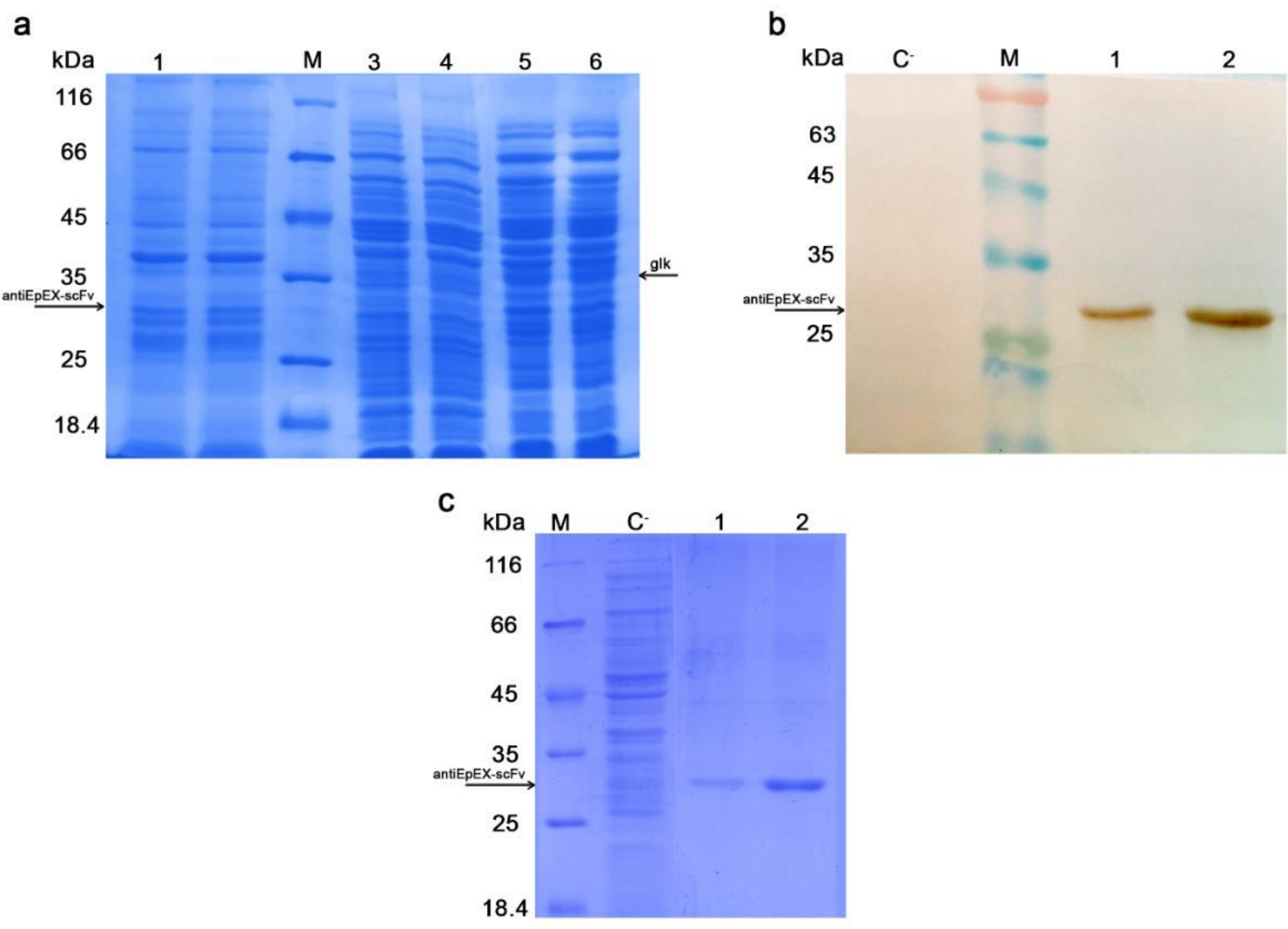

\section{Figure 5}

Growth profiles and glucose consumption rates. When OD600 reached to 0.8 , cells were induced for $24 \mathrm{~h}$ using $0.8 \mathrm{mM} \mathrm{IPTG}$. Growth and glucose consumption profiles of the wild type (BW25113) and the recombinant strains in $\mathrm{M} 9$ medium supplemented with $5 \mathrm{~g} / \mathrm{L}$ glucose (A, B) and $10 \mathrm{~g} / \mathrm{L}$ glucose (C, D). Error bars illustrated the standard deviation of two experimental replicates. All graphs are drawn using GraphPad Prism 8 software. Data are presented as mean \pm SD, $n=2$. 